\title{
Hamiltonian Transition Matrices
}

\author{
Konstantin Avrachenkov \\ INRIA Sophia Antipolis \\ France \\ K.Avrachenkov@inria.fr
}

\author{
Ali Eshragh \\ School of Mathematics and \\ Statistics \\ University of South Australia \\ Ali.Eshragh@unisa.edu.au Jerzy.Filar@unisa.edu.au \\ Jerzy A. Filar \\ School of Mathematics and \\ Statistics \\ University of South Australia \\ Jerzy.Filar@unisa.edu.au
}

\begin{abstract}
In this pedagogical note, we present some algebraic properties of a particular class of probability transition matrices, namely, Hamiltonian transition matrices. Each matrix $P$ in this class corresponds to a Hamiltonian cycle in a given graph $G$ on $n$ nodes and to an irreducible, periodic, Markov chain. We show that a number of important matrices traditionally associated with Markov chains, namely, the stationary, fundamental, deviation and the hitting time matrix all have elegant expansions in the first $n-1$ powers of $P$, whose coefficients can be explicitly derived. We also consider the resolvent-like matrices associated with any given Hamiltonian cycle and its reverse cycle and prove an identity about the product of these matrices.
\end{abstract}

\section{Categories and Subject Descriptors}

G.2.2 [Discrete Mathematics]: Graph Theory-Path and Circuit Problems; G.3 [Probability and Statistics]: Markov Processes

\section{General Terms}

Theory

\section{Keywords}

Hamiltonia Path Problem, Hamiltonian Transition Matrices, Markov Chains

\section{INTRODUCTION}

One of the central concepts in graph theory is the Hamiltonian cycle. Given a graph $G$, a simple path that starts from one node, visits all nodes exactly once and returns to the initial node is called a Hamiltonian cycle or a tour. It should be noted that in this context, terms "Hamiltonian cycle" and "tour" are used, interchangeably. Accordingly, we can define the Hamiltonian Cycle Problem (HCP), which is a well-known problem in graph theory. Particularly, given a directed graph $G$, we are asked to determine whether it contains at least one tour or not. If $G$ contains at least one tour,

Permission to make digital or hard copies of all or part of this work for personal or classroom use is granted without fee provided that copies are not made or distributed for profit or commercial advantage and that copies bear this notice and the full citation on the first page. To copy otherwise, to republish, to post on servers or to redistribute to lists, requires prior specific permission and/or a fee.

VALUETOOLS 2011, May 16-20, Paris, France

Copyright @ 2011 ICST 978-1-936968-09-1

DOI 10.4108/icst.valuetools.2011.245841 then the graph is called Hamiltonian and otherwise, that is, if there exists no tour in $G$, it is called a non-Hamiltonian graph. In spite of its simple appearance, HCP is an NPcomplete problem [4, Chapter 3].

One of the approaches to tackle this problem was proposed in Filar and Krass [2], where the deterministic Hamiltonian cycle problem is converted to a particular average-reward Markov decision process. That paper, was the motivation of a new line of research for the HCP that was summarized in [3]. Essential in this approach are the expansions of key matrices such as the stationary and fundamental matrices associated with various Markov chains induced by the graph $G$ under consideration. Of course, in general, these expansions are infinite series. However, if we restrict ourselves to probability transition matrices induced by Hamiltonian cycles, the preceding expansions reduce to elegant finite series with easily computable coefficients.

The bulk of this pedagogical note is devoted to collecting and deriving the above series expansions with the help of Markov chains techniques. To the best of our knowledge the explicit forms of all these series - with closed form expressions for their coefficients - have not been reported elsewhere. In addition, we derive (by similar techniques) an apparently novel identity for the product of the resolvent-like matrices associated with a Hamiltonian cycle and its reverse. Recall that, in an undirected graph, every Hamiltonian cycle is accompanied by a reverse cycle whose probability transition matrix is simply the transpose of that corresponding to the original cycle.

We omit all the proofs which will appear in a journal version of the present work.

\section{HAMILTONIAN TRANSITION MATRI- CES}

Consider a given labeled graph $G$ on $n$ nodes. Suppose $\mathcal{V}=$ $\{1,2, \ldots, n\}$ and $\mathcal{A}$ are, respectively, sets of all nodes and arcs in graph $G$. If $G$ is Hamiltonian, then corresponding to each tour $\tau$ in $G$, we can construct a probability transition matrix, namely Hamiltonian transition matrix.

Definition 2.1. Suppose the Hamiltonian graph $G$ is given and $\tau$ is a tour in $G$. We say the probability transition matrix $P$ is "Hamiltonian transition matrix", if it is a $0-1$ matrix such that its positive elements trace out tour $\tau$ in $G$. 
For instance, the components of the Hamiltonian transition matrix $^{1} P$ associated with the standard tour " $1 \rightarrow 2 \rightarrow$ $\cdots \rightarrow n \rightarrow 1 "$ is

$$
p_{i j}=\left\{\begin{array}{ll}
1 & \text { for } i=1,2, \ldots, n-1, j=i+1 \\
1 & \text { for } i=n, j=1 \\
0 & \text { otherwise }
\end{array} .\right.
$$

Of course, Hamiltonian matrices can also be seen as special permutation matrices but, in this note, it is their nature as special probability transition matrices of Markov chains that we focus on. Undoubtedly, results we present here could also be derived by other methods that do not exploit Markov chain interpretations but matrices such as the fundamental, deviation and stationary distribution matrix are, perhaps, of most interest to researchers using Markov chains as modelling tools.

It should be noted that in all subsequent sections, we suppose that $P$ is a Hamiltonian matrix induced by a tour in the Hamiltonian graph $G$ on $n$ nodes, unless, otherwise stated. Also, we adopt the convention that matrix $A^{T}$ denotes the transpose of the matrix $A$.

We begin by recalling, a number of obvious properties of Hamiltonian matrices that follow directly definitions and Chapman-Kolmogorov equations. These are summarised in the following lemma.

Lemma 2.2. Suppose that the Hamiltonian matrix $P$ is induced by the tour $\tau:$ : $\ell_{0}(=i) \rightarrow \ell_{1} \rightarrow \cdots \rightarrow \ell_{n-1} \rightarrow$ $\ell_{0} "$.

(i) For values of $r=0,1, \ldots, n-1$, the $i j^{\text {th }}$ component of matrix $P^{r}$ will be equal to

$$
p_{i j}^{(r)}=\delta_{\ell_{r} j},
$$

where, $\delta$ is the Kronecker's delta.

(ii) The Hamiltonian matrix $P$ has period $n$ and, more generally

$$
\begin{gathered}
P^{k n+r}=P^{r} \quad \text { for } k=0,1,2, \ldots \\
\text { and } r=0,1,2, \ldots, n-1
\end{gathered}
$$

(iii) The eigenvalues of $P$ are the $n$ roots of unity.

\section{MARKOV CHAINS}

According to Definition 2.1, any Hamiltonian matrix is a probability transition matrix. This implies that we can derive all properties of probability transition matrices for them, as well.

REMARK 3.1. According to Lemma 2.2, for each pair of $i, j \in \mathcal{V}$, we have $p_{i j}^{(r)}>0$ for some $r \in\{0,1, \ldots, n-1\}$. This implies that all states in $\mathcal{V}$ communicate with each other and consequently, the Markov chain defined by each Hamiltonian matrix is irreducible.

\footnotetext{
${ }^{1}$ Henceforth, for simplicity, we call Hamiltonian transition matrices just as Hamiltonian matrices.
}

Remark 3.1 indicates that for each Hamiltonian matrix, there exists a unique stationary distribution, as well as, Ceasro limit matrix. In the following two lemmas, we derive an explicit equation for each of them.

LEMMA 3.2. The stationary distribution of the Hamiltonian matrix $P$, say $\boldsymbol{\pi}$, follows the uniform distribution.

\section{Proposition 3.3. For Hamiltonian matrix P,}

(i) the Cesaro limit matrix, say $P^{*}$, is equal to $\frac{1}{n} J$, where $J$ is a square matrix with all entries equal to 1 ;

(ii) matrix $P^{*}$ can be expressed in a finite sum of powers of $P$ as follows

$$
P^{*}=\frac{1}{n} \sum_{r=0}^{n-1} P^{r}
$$

One may be interested in defining the (first) hitting time matrix $H$, such that its $i j^{t h}$ component, $h_{i j}$, identifies the expected hitting time of node $j$, starting from node $i$ and following Markov chain defined by the Hamiltonian matrix $P$. From Lemma 2.2 , it is readily seen that

$$
h_{i j}=\min _{0 \leq r \leq n-1}\left\{r \mid p_{i j}^{(r)}>0\right\} .
$$

Accordingly, we can express the hitting time matrix in terms of the first $n$ powers of $P$.

Lemma 3.4. For a Hamiltonian matrix $P$, the hitting time matrix can be expressed in terms of the first $n$ powers of $P$ as follows

$$
H=\sum_{r=0}^{n-1} r P^{r}
$$

Surprisingly, irrespective of the Hamiltonian cycle that defines matrix $P$, we can show that the inverse of corresponding hitting time matrix $H$ can be expressed in sum of finite powers of $P$, as well. For this purpose, firstly, we need to show the following lemma.

Lemma 3.5. Consider the Hamiltonian matrix $P$ and its corresponding hitting time matrix $H$. We have

(i) $H P=H-J+n I$;

(ii) $H J=\frac{n(n-1)}{2} J$.

Proposition 3.6. Consider the Hamiltonian matrix $P$ and its corresponding hitting time matrix $H$. The inverse of matrix $H$ exists and can be expressed as a finite sum of powers of matrix $P$ as follows:

$$
H^{-1}=-\frac{n^{2}-n-2}{n^{2}(n-1)} I+\frac{n^{2}-n+2}{n^{2}(n-1)} P+\frac{2}{n^{2}(n-1)} \sum_{r=2}^{n-1} P^{r} .
$$




\section{MATRICES ASSOCIATED WITH MARKOV CHAINS}

In this section, we, mainly, want to derive explicit formulation for classical matrices in Markov decision processes induced by Hamiltonian matrices. At the outset, we recall such matrices from [5, Appendix A]. It must be noted that following definitions hold not only for Hamiltonian matrices, but also for any probability transition matrix $P$.

The fundamental matrix is denoted by $G$ and defined as follows

$$
F:=\left(I-P+P^{*}\right)^{-1},
$$

where, $P$ is a probability transition matrix and $P^{*}$ is its Cesaro limit matrix. Accordingly, we can define the deviation matrix through

$$
D:=F-P^{*} \text {. }
$$

The main properties of these matrices asserted in the following theorem (e.g., see [5, Appendix A]):

THEOREM 4.1. If $P$ is a probability transition matrix with Cesaro limit matrix $P^{*}$, then it is well-known that: $(i) P^{* 2}=$ $P^{*}$; $($ ii $) P P^{*}=P^{*} P=P^{*} ;($ iii $)\left(P-P^{*}\right)^{k}=P^{k}-P^{*}$ for $k=$ $1,2, \ldots ;\left(\right.$ iv) $F P^{*}=P^{*} F=P^{*} ;(v) D P^{*}=P^{*} D=0$.

Based on mentioned definitions and notations, we want to know if we can express the fundamental matrix, as well as, the deviation matrix induced by a Hamiltonian matrix $P$ as a finite sum of powers of $P$.

Proposition 4.2. For a Hamiltonian matrix $P$, the corresponding fundamental matrix $F$ and deviation matrix $D$, can be expressed as follows:

$$
\begin{aligned}
& F=\sum_{r=0}^{n-1} \frac{n+1-2 r}{2 n} P^{r} \\
& D=\sum_{r=0}^{n-1} \frac{n-1-2 r}{2 n} P^{r} .
\end{aligned}
$$

Corollary 4.3. If $P$ is a Hamiltonian matrix, then the $i j^{\text {th }}$ component of its corresponding fundamental matrix $F$ is equal to

$$
\frac{n+1-2 h_{i j}}{2 n}
$$

REMARK 4.4. Borkar et. al. [1] exploited the optional sampling theorem to develop explicit formulae for entries of a fundamental matrix $F$ induced by a doubly stochastic matrix ${ }^{2}$ $P$. More precisely, they expressed each entry of $F$ in terms of expected hitting times. As each Hamiltonian matrix is also a doubly stochastic matrix, naturally, (8) coincides with results in [1].

\footnotetext{
${ }^{2}$ A matrix is called doubly stochastic, if its entries are nonnegative, as well as, all rows and columns sum up to 1 .
}

Corollary 4.5. For a given Hamiltonian matrix, the powers of corresponding fundamental matrix as well as deviation matrix can be expressed as a sum of the first $n$ powers of matrix $P$, that is,

$$
\begin{aligned}
F^{k} & =\sum_{r=0}^{n-1} f_{r}^{k} P^{r} \quad \text { for } k=1,2, \ldots \\
D^{k} & =\sum_{r=0}^{n-1} d_{r}^{k} P^{r} \quad \text { for } k=1,2, \ldots,
\end{aligned}
$$

where, $f_{r}^{k}$ and $d_{r}^{k}$ are real coefficients.

Now, one may be interested in finding coefficients $f_{r}^{k}$ and $d_{r}^{k}$. Obviously, from Proposition 4.2, we know that,

$$
f_{r}^{1}=\frac{n+1-2 r}{2 n} ; \quad d_{r}^{1}=\frac{n-1-2 r}{2 n},
$$

for $r=0,1, \ldots, n-1$. Hence, if we can find a recursive formulation for them, we will be able to calculate them, directly.

Proposition 4.6. For a given Hamiltonian matrix $P$ and its corresponding fundamental matrix, the following hold for $k=1,2, \ldots$ :

(i) $\sum_{r=0}^{n-1} f_{r}^{k}=1$,

(ii) $\left\{\begin{array}{l}f_{0}^{k+1}=f_{0}^{k}+\frac{1}{n} \sum_{s=1}^{n-1} s f_{s}^{k}-\frac{n-1}{2 n} \\ f_{r}^{k+1}=f_{0}^{k+1}+\sum_{s=1}^{r} f_{s}^{k}-\frac{r}{n} \text { for } r=1,2, \ldots, n-1\end{array}\right.$

In order to derive analogous recursive formula for coefficients $d_{r}^{k}$, we first find a linear relationship between coefficients $d_{r}^{k}$ and $f_{r}^{k}$, and then, exploit Proposition 4.6.

Lemma 4.7. For a given Hamiltonian matrix $P$ and the corresponding fundamental and deviation matrices, we have

$$
d_{r}^{k}=f_{r}^{k}-\frac{1}{n} \text { for } r=0,1, \ldots, n-1, k=1,2, \ldots
$$

COROLlary 4.8. For a given Hamiltonian matrix $P$ and the corresponding deviation matrix, the following hold for $k=1,2, \ldots$ :

(i) $\sum_{r=0}^{n-1} d_{r}^{k}=0$,

(ii) $\left\{\begin{array}{l}d_{0}^{k+1}=d_{0}^{k}+\frac{1}{n} \sum_{s=0}^{n-1} s d_{s}^{k} \\ d_{r}^{k+1}=d_{0}^{k+1}+\sum_{s=1}^{r} d_{s}^{k} \text { for } r=1,2, \ldots, n-1\end{array}\right.$. 
Another matrix that plays an essential role in the theory of discounted Markov decision processes we shall name the $\beta$-resolvent matrix. More precisely, if $P$ is a probability transition matrix and $\beta \in(0,1)$ is a discount factor, the $\beta$-resolvent matrix, $R(\beta)$, is defined as follows:

$$
R(\beta):=(I-\beta P)^{-1} \text {. }
$$

Analogous to the fundamental and deviation matrices, for the special case of Hamiltonian matrix $P$, we can derive a finite sum of powers of matrix $P$ for $R(\beta)$, as expressed in the following proposition.

Proposition 4.9. If $P$ is a Hamiltonian matrix, then for any value of discount factor $\beta \in(0,1)$, we will have

$$
R(\beta)=\frac{1}{1-\beta^{n}} \sum_{r=0}^{n-1} \beta^{r} P^{r} .
$$

Corollary 4.10. If $P$ is a Hamiltonian matrix, the $i j^{\text {th }}$ component of its $\beta$-resolvent matrix $R(\beta)$ is equal to

$$
\frac{\beta^{h_{i j}}}{1-\beta^{n}}
$$

\section{UNDIRECTED GRAPHS}

A large subclass of all graphs is the class of undirected graphs. Apparently, if a given undirected graph $G$ is Hamiltonian, then corresponding to each tour $\tau:$ " $\ell_{0} \rightarrow \ell_{1} \rightarrow \cdots \rightarrow$ $\ell_{n-1} \rightarrow \ell_{0}$ " in $G$, its reverse, $\tau^{\mathcal{R}}:$ " $\ell_{0} \rightarrow \ell_{n-1} \rightarrow \cdots \rightarrow$ $\ell_{1} \rightarrow \ell_{0}$ ", also exists in $G$. Accordingly, if the Hamiltonian matrix $P$ is corresponding to tour $\tau$, then its transpose, $P^{T}$ is corresponding to the reverse tour $\tau^{\mathcal{R}}$. Hence, the following result can be obtained, immediately. It should be noted that in this section, our results concern undirected graphs, unless otherwise is mentioned.

Lemma 5.1. For a Hamiltonian matrix $P, P P^{T}=I$.

Corollary 5.2. For a Hamiltonian matrix $P$,

$$
\left(P^{T}\right)^{r}=P^{n-r} \text { for } r=0,1, \ldots, n-1 .
$$

We can also derive the following result for hitting time matrix in an undirected graph.

Lemma 5.3. Consider the Hamiltonian matrix $P$ corresponding to tour $\tau$ and its corresponding hitting time matrix $H$. If $H^{\mathcal{R}}$ denotes the hitting time matrix associated with the reverse tour $\tau^{\mathcal{R}}$, we will have

(i) $H^{\mathcal{R}}=\sum_{r=1}^{n-1}(n-r) P^{r}$;

(ii) $H+H^{\mathcal{R}}=n(J-I)$.
Consider the Hamiltonian matrix $P$ and its corresponding $\beta$-resolvent matrix $R(\beta)$. Let $R^{\mathcal{R}}(\beta)$ denote the $\beta$-resolvent matrix associated with the reverse tour corresponding to $P$, that is, $R^{\mathcal{R}}(\beta)=\left(I-\beta P^{T}\right)^{-1}$. The following proposition expresses an interesting relationship between them. However, we need the following lemma to prove it, in advance.

Lemma 5.4. For a Hamiltonian matrix $P$, the $i j^{\text {th }}$ component of its corresponding matrix $R^{\mathcal{R}}(\beta)$ is equal to

$$
r_{i j}^{\mathcal{R}}(\beta)=\frac{\beta^{h_{j i}}}{1-\beta^{n}} \text {. }
$$

Proposition 5.5. If $P$ is a Hamiltonian matrix corresponding to a tour $\tau$, then for any value of discount factor $\beta \in(0,1)$, we will have

$$
R(\beta) R^{\mathcal{R}}(\beta)=\frac{1}{1-\beta^{2}}\left(R(\beta)+R^{\mathcal{R}}(\beta)-I\right) .
$$

\section{CONCLUSION}

In this paper, we presented algebraic properties of a particular class of probability transition matrices, namely, Hamiltonian matrices. In this class, each matrix corresponds to a Hamiltonian cycle in a given graph $G$ on $n$ nodes. We showed that such matrices are periodic with periodicity equal to $n$. We considered several famous matrices in the context of Markov chains and derive them as a convex combination of the first $n$ powers of corresponding Hamiltonian matrix $P$.

\section{REFERENCES}

[1] V.S. Borkar, V. Ejov and J. Filar, "On the Hamiltonicity Gap and Doubly Stochastic Matrices", Random Structures \& Algorithms, 34(4):502-519, 2009.

[2] J.A. Filar and D. Krass, "Hamiltonian Cycles and Markov Chains", Mathematics of Operations Research, 19(1):223-237, 1994.

[3] J.A. Filar, "Controlled Markov Chains, Graphs \& Hamiltonicity", Foundation and Trends ${ }^{\circledR}$ in Stochastic Systems, 1(2):77-162, 2006.

[4] M.R. Garey and D.S. Johnson, Computers and Intractability: A Guide to the Theory of NP-Completeness, W.H. Freeman \& Co., 1979.

[5] M.L. Puterman, Markov Decision Processes: Discrete Stochastic Dynamic Programming, Wiley-Interscience, $1^{\text {st }}$ Edition, 2005. 